\title{
Composição florística e estrutural de um componente arbóreo em área de cerrado, Paranã-TO
}

\section{Floristic composition and structural components of a tree in closed area in the municipality of Parana-Tocantins}

\author{
Andreia Brandão de Melo Lopes Rêgo ${ }^{1}$, Priscila Bezerra de Souza ${ }^{2}$, Rubens Ribeiro da Silva ${ }^{3}$, Pietro Lopes Rêgo ${ }^{4}$
}

Resumo: O Cerrado Brasileiro apresenta uma enorme diversidade de fitofisionomias divididas em formações florestais, savânicas e campestres. Com o objetivo de estudar a composição florística e estrutural de um fragmento de cerrado sensu stricto, a fim de fornecer subsídios para conservação e manejo adequado de áreas similares. Foram instaladas sistematicamente quatro parcelas de $25 \times 100 \mathrm{~m}$, sendo que as mesmas foram distanciadas $25 \mathrm{~m}$ entre si, perfazendo um total de 1,0 hectare de área amostral. No interior das parcelas, foram amostrados todos os indivíduos arbustivo-arbóreos, com circunferência a 1,30m do solo (CAP) maior ou igual a $15 \mathrm{~cm}$. Para obtenção dos parâmetros fitossociológicos densidade relativa (DR), densidade absoluta (DA), dominância absoluta (DoA), dominância relativa (DoR), frequência absoluta (FA), frequência relativa (FR), índice de valor de importância (IVI), índice de valor de cobertura (IVC), índice de diversidade de Shannon (H') e Pielou (J). Foram amostrados 245 indivíduos, distribuídos em 29 espécies, 29 gêneros pertencentes a 20 famílias, o que corresponde a uma área basal total de $1,94 \mathrm{~m}^{2}$.ha ${ }^{-1}$ e densidade absoluta estimada de 327 ind.ha ${ }^{-1}$. As espécies que apresentaram maiores valores de (IVI) foram Qualea parviflora (38,45\%), Curatella americana (34,53\%), Byrsonima verbascifolia (32,20\%), Psidium incanescens (31,89\%), Pterodon emarginatus (18,62\%) e Anachardium humile (18,34\%). Qualea parviflora foi a espécie mais representativa dentro do componente arbóreo o que se conclui aos altos valores de densidade e dominância nos estratos.

Palavras-chave: Fitossociologia. Distribuição diamétrica. Distribuição hipsométrica

\begin{abstract}
The Brazilian Cerrado presents a huge diversity of vegetation types divided into forests, savannas and country. In order to study the floristic and structural composition of a cerrado fragment in order to provide subsidies for conservation and proper management of similar areas. Systematically they were installed four installments of $25 \times 100 \mathrm{~m}$, being that they were spaced $25 \mathrm{~m}$ apart, totaling 1.0 hectare sample area. Inside the plots were sampled every shrub and individual trees with circumference at $1.30 \mathrm{~m}$ soil (CAP) greater than or equal to $15 \mathrm{~cm}$. To obtain the Phytosociological relative density parameters (DR), absolute density (DA), absolute dominance (DoA), relative dominance (DoR), absolute frequency (AF), relative frequency (RF), importance value index (IVI) Coverage value index (CVI), Shannon diversity index (H ') and Pielou (J). We sampled 245 individuals belonging to 29 species, 29 genera belonging to 20 families, which corresponds to a basal area of 1.94 $\mathrm{m}^{2} \cdot \mathrm{ha}^{-1}$ and estimated absolute density of 327 ind.ha $^{-1}$. The species showing higher values of (IVI) were Qualea parviflora (38.45\%), American Curatella (34.53\%), Byrsonima verbascifolia (32.20\%), Psidium incanescens (31.89\%), Pterodon emarginatus (18.62\%) and Anachardium humile (18.34\%). Qualea parviflora was the most representative species within the arboreal component which is concluded to high density values and dominance in the strata.
\end{abstract}

Key words: Phytosociology. Diametric hypsometric. Diametric distribution.

\footnotetext{
*Autor para correspondência

Recebido para publicação em 14/11/2015; aprovado em 28/12/2015

${ }^{1}$ Mestranda do Programa de Pós-graduação em Ciências Florestais e Ambientais - Campus Gurupi/UFT. E-mail: andreia.b.melo@ @otmail.com

${ }^{2}$ Prof. ${ }^{a}$ Dr. ${ }^{a}$ da disciplina Ecologia do Cerrado do Programa de Pós-graduação em Ciências Florestais e Ambientais - Campus Gurupi/UFT.

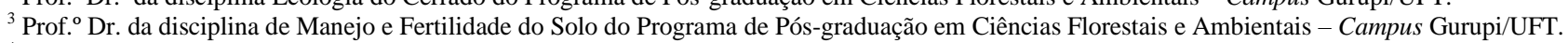

${ }^{4}$ Prof. ${ }^{o}$ M.Sc., do Campus Dianópolis - Instituto Federal de Educação, Ciência e Tecnologia do Tocantins - IFTO.
} 


\section{INTRODUÇÃO}

O Cerrado é o segundo maior bioma da América do Sul, abrangendo uma área de $2.036 .448 \mathrm{~km}^{2}$, cerca de $22 \%$ do território nacional, ocupando o Planalto Central do Brasil. Neste espaço territorial encontram-se as nascentes das três maiores bacias hidrográficas da América do Sul (Amazônica/Tocantins, São Francisco e Prata), o que resulta em um elevado potencial aquífero e favorece a sua biodiversidade (BRASIL, 2014).

É considerado um dos 25 "hotspots" mundiais de biodiversidade, em função de sua riqueza biótica, nível de endemismos e grau de ameaça (MITTERMEIER et al. 1999; MYERS et al. 2000). Porém, somente a partir da década de 1980 que seus aspectos florísticos e ecológicos passaram a ser alvo de investigação científica sistemática.

Diversos levantamentos florísticos e fitossociológicos têm fornecido informações importantes para a compreensão dos padrões biogeográficos do Cerrado, e subsidiado a determinação de áreas prioritárias para a conservação (FELFILI et al., 2002; SILVA et al., 2002; PEREIRA-SILVA et al., 2004). Considerando que a fisionomia representa uma característica vegetacional facilmente mensurável, os estudos sobre a composição florística e a ecologia das comunidades vegetais são fundamentais para embasar quaisquer iniciativas de preservação e conservação dos remanescentes florestais. Além de ser uma importante ferramenta para subsidiar o desenvolvimento de modelos de recuperação de áreas degradadas, ou seja, para a seleção de espécies para fins silviculturais e utilização racional dos recursos vegetais (OLIVEIRA-FILHO et al., 2004).

Diversos autores têm pesquisado o efeito de fatores como: idade, sítio, densidade e posição sociológica sobre a dinâmica da distribuição diamétrica de diferentes espécies. É sabido que o crescimento acumulativo em diâmetro tende a aumentar com o avançar da idade, melhoria do sítio, porém tende a reduzir-se com o aumento da densidade, seja ela inicial, seja presente (MACHADO et al., 2006).

As diferentes formações florestais apresentam distribuições diamétricas diferentes, tanto na sua amplitude, como na sua forma. Por isso, a distribuição de diâmetro é característica importante para a avaliação de estoque em crescimento (FERREIRA et al., 1998). Segundo Siminski et al. (2004), a distribuição diamétrica busca permitir a avaliação prévia de condições dinâmicas da floresta, possibilitando previsões futuras quanto ao desenvolvimento da comunidade vegetal. A distribuição diamétrica pode ser entendida como mais um elemento do componente estrutura horizontal do povoamento.

O conhecimento sobre a distribuição e organização da biodiversidade, nas fisionomias do domínio Cerrado ainda são escassos. Entretanto estas informações são de grande importância para avaliar os impactos antrópicos, planejar a criação de unidades de conservação além da adoção de técnicas de manejo (FELFILI; SILVA JÚNIOR, 2001).

Em virtude da elevada biodiversidade das formações florestais neotropicais, da variação nas classes de diâmetro e condições ecofisiológicas da grande diversidade de espécies objetivou-se estudar a composição florística e estrutural de um fragmento de cerrado sensu stricto, a fim de fornecer subsídios para conservação e manejo adequado de áreas similares

\section{MATERIAL E MÉTODOS}

\section{Área de estudo}

$\mathrm{O}$ estudo foi desenvolvido em um fragmento de cerrado sensu stricto inserido numa propriedade particular Fazenda Retiro III de aproximadamente 456,48 hectares, sob as coordenadas geográficas $12^{\circ} 33^{\prime} 60^{\prime}$ ' $\mathrm{S}$ e $47^{\circ} 59^{\prime} 12^{\prime \prime} \mathrm{W}$, município de Paranã, região sudeste do Estado do Tocantins.

O clima da região segundo o sistema de ThornthwaiteMather é do tipo B2rA'a' -clima úmido com pequena ou nula deficiência hídrica. A temperatura média ao longo do ano varia entre $22^{\circ} \mathrm{C}$ e $36^{\circ} \mathrm{C}$ já a precipitação média anual varia entre $1200 \mathrm{~mm}$ a $1300 \mathrm{~mm}$. A umidade relativa é elevada, sendo a média real de 78\% (MARCUZZO et. al., 2011).

Os solos da região apresentam manchas de Gleissolos e Plintossolo, sendo que a topografia do fragmento apresenta níveis altimétricos variados, originado de um relevo colinoso existente na área com seus níveis oscilando entre 150 e 320 metros (SEPLAN-TO, 2015).

\section{Metodologia de amostragem}

A vegetação na área estudada foi avaliada pelo o método de parcela (MUELLER-DOMBOIS; ELLENBERG, 1974). Foram instaladas sistematicamente quatro parcelas de $25 \times 100 \mathrm{~m}^{2}$, sendo que as mesmas foram distanciadas $25 \mathrm{~m}$ entre si, perfazendo um total de 1,0 hectare de área amostral. No interior das parcelas, foram amostrados todos os indivíduos arbustivo-arbóreos, com circunferência a 1,30m do solo (CAP) maior ou igual a $15 \mathrm{~cm}$.

A identificação taxonômica das espécies foi realizada em campo, caso contrário o material botânico foi coletado para posterior identificação por meio de comparações com o material do Herbário da UFT, campus de Porto Nacional, literatura especializada e consultas a especialistas. A utilização de binômios específicos foi feita baseada no índice de espécies da Royal Botanic Gardensof Kewe o site do Missouri Botanical Garden (disponível em: http://www.mobot.org/wt3/search/vast.html). O sistema de classificação adotado foi o "Angiosperm Phylogeny Group" (APG III, 2009).

\section{Análise dos dados}

Para obtenção dos parâmetros fitossociológicos densidade relativa (DR), densidade absoluta (DA), dominância absoluta (DoA), dominância relativa (DoR), frequência absoluta (FA), frequência relativa (FR), índice de valor de importância (IVI), índice de valor de cobertura (IVC), índice de diversidade de Shannon (H') e Pielou (J), ambos os índices foram calculados pelas formas tradicionais, a partir do uso do software Excel 2010.

Os dados básicos, obtidos das quatro parcelas, foram analisados para fins de obtenção da matriz que relaciona o número de árvores, por hectare, da i-ésima espécie na j-ésima classe de diâmetro. Os indivíduos amostrados foram distribuídos em 4 classes de diâmetro de $5 \mathrm{~cm}$ de amplitude (FERREIRA, 1988).

Para analisar a estrutura hipsométrica foi confeccionado um histograma relacionando o número de indivíduos por classe de altura com o intervalo de $1 \mathrm{a} 4 \mathrm{~m}$, para a estrutura diamétrica o histograma foi correlacionado com o número de indivíduos por classe de diâmetro com seus respectivos intervalos, além disso foi construído um histograma 
correlacionando área basal com as classes diámetricas. Para a obtenção das tabelas e gráficos de distribuição diamétrica, empregou-se os software Microsoft Office Excel 2010.

\section{RESULTADOS E DISCUSSÃO}

Foram amostrados 245 indivíduos, distribuídos em 29 espécies, 29 gêneros pertencentes a 20 famílias, o que corresponde a uma área basal total de $1,94 \mathrm{~m}^{2} \cdot \mathrm{ha}^{-1} \mathrm{e}$ densidade absoluta estimada de 327 ind.ha $^{-1}$.

$O$ índice de diversidade de Shannon Weaver ( $\left.\mathrm{H}^{\prime}\right)$ encontrado foi de 2,60 nats/ind., valor próximo aos comumente encontrados em áreas de cerrado sensu stricto. Entretanto, apesar deste índice encontrado, muitas espécies estão representadas por um ou poucos indivíduos, o que não garante sua sustentação no fragmento, em que Brito et al. (2006) em estudo na região sudoeste do estado encontrou diversidade de 3,44 nats/ind.

Das 20 famílias amostradas, quatro corresponderam por $65 \%$ do VI das dez espécies mais representativas do fragmento, Vochysiaceae (3), Dilleniaceae (1), Malpighiaceae (1) e Myrtaceae (2).

Analisando a flora como um todo, as famílias com maior riqueza de espécies encontradas neste levantamento também são as mais ricas no bioma Cerrado segundo (MENDONÇA et al. 1998)

As espécies que apresentaram maiores valores de (IVI) foram Qualea parviflora (38,45\%), Curatella americana (34,53\%), Byrsonima verbascifolia (32,20\%), Psidium incanescens $(31,89 \%)$, Pterodon emarginatus $(18,62 \%)$ e Anachardium humile (18,34\%).

As espécies que se destacaram em valores de freqüência relativa foram Qualea parviflora, Curatella americana, Byrsonima verbascifolia, Psidium incanescens, Pterodon emarginatus, Anachardium humile, Pseudobombax grandiflorum e Andira cuyabensis totalizando 5,36\%. Das 29 espécies encontradas, apenas 8 estiveram presentes em todas as parcelas. Isto evidencia uma distribuição irregular das espécies no fragmento, que possui poucas espécies uniformemente presentes na área amostrada e pequeno número de espécie por unidade de área.

Tabela 1. Parâmetros fitossociológicos das espécies amostradas em 1,0 ha de cerrado sensu stricto, em ordem decrescente de $\mathrm{VI}=$ valor de importância (\%); DA= densidade absoluta (número de indivíduos/ha); $\mathrm{N} \mathrm{Sp}=$ número de espécies; \% Sp = porcentagem de espécies; $\mathrm{DR}=$ densidade relativa $(\%)$; DoA = dominância absoluta $(\%)$; DoR = dominância relativa $(\%)$; FA= frequência absoluta $(\%)$ e $\mathrm{FR}=$ frequência relativa $(\%)$.

\begin{tabular}{|c|c|c|c|c|c|c|c|c|c|}
\hline Espécies & Nome Comum & n.i & $\begin{array}{c}\text { F.A. } \\
\%\end{array}$ & $\begin{array}{c}\text { F.R. } \\
\%\end{array}$ & $\begin{array}{c}\text { D.A. } \\
\text { ind.ha }^{-1}\end{array}$ & $\begin{array}{l}\text { D.R. } \\
\%\end{array}$ & $\frac{\text { Do.A. }}{\text { m }^{2}}$ & $\frac{\text { Do.R. }}{\%}$ & V.I. \\
\hline Qualea parvifloraMart. & Pau terra & 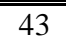 & 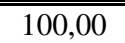 & 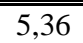 & 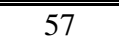 & 17,55 & 0,40 & 15,54 & 38,45 \\
\hline Curatella americana $\mathrm{L}$ & Simbaíba & 40 & 100,00 & 5,36 & 53 & 16,33 & 0,33 & 12,85 & 34,53 \\
\hline $\begin{array}{l}\text { Byrsonima verbascifolia }(\mathrm{L} .) \text { Rich. ex } \\
\text { Juss. }\end{array}$ & Murici & 35 & 100,00 & 5,36 & 47 & 14,29 & 0,33 & 12,56 & 32,20 \\
\hline Psidium incanescensMart. ex DC. & Araçá & 37 & 100,00 & 5,36 & 49 & 15,10 & 0,30 & 11,43 & 31,89 \\
\hline Pterodon emarginatus Vogel. & Sucupira branca & 11 & 100,00 & 5,36 & 15 & 4,49 & 0,23 & 8,77 & 18,62 \\
\hline Anachardium humileST. HILL & Caju do campo & 13 & 100,00 & 5,36 & 17 & 5,31 & 0,20 & 7,68 & 18,34 \\
\hline $\begin{array}{l}\text { Pseudobombax grandiflorum(Cav.) } \\
\text { A.Robyns }\end{array}$ & $\begin{array}{l}\text { Embiruçu do } \\
\text { cerrado }\end{array}$ & 4 & 100,00 & 5,36 & 5 & 1,63 & 0,09 & 3,47 & 10,46 \\
\hline $\begin{array}{l}\text { Andira cuyabensisL.C. Bernacci \& } \\
\text { G.F. Árbocz }\end{array}$ & $\begin{array}{l}\text { Angelim do } \\
\text { cerrado }\end{array}$ & 6 & 100,00 & 5,36 & 8 & 2,45 & 0,06 & 2,48 & 10,29 \\
\hline Salvertia convallariaeodoraSt.Hill & Bananeira & 4 & 66,67 & 3,57 & 5 & 1,63 & 0,10 & 3,70 & 8,90 \\
\hline Bagassa guianensisAubl. & Garroteiro & 5 & 66,67 & 3,57 & 7 & 2,04 & 0,08 & 3,04 & 8,65 \\
\hline Caesalpinia pyramidalisTul. & Pau de rato & 4 & 66,67 & 3,57 & 5 & 1,63 & 0,08 & 2,93 & 8,13 \\
\hline Styrax ferrugineus Nees \& Mart. & Pindaíba & 7 & 66,67 & 3,57 & 9 & 2,86 & 0,04 & 1,51 & 7,94 \\
\hline Eugenia dysenterica DC & Cagaita & 6 & 66,67 & 3,57 & 8 & 2,45 & 0,05 & 1,91 & 7,93 \\
\hline Astronium flaxinifolium Schott. & Gonçalo Alves & 5 & 66,67 & 3,57 & 7 & 2,04 & 0,04 & 1,39 & 7,00 \\
\hline Dalbergia miscolobiumBenth. & Jacarandá & 2 & 66,67 & 3,57 & 3 & 0,82 & 0,06 & 2,18 & 6,57 \\
\hline Drimys winteriJ.R.Forst. \& G.Forst. & Casco d'anta & 3 & 66,67 & 3,57 & 4 & 1,22 & 0,04 & 1,40 & 6,19 \\
\hline Cariniana estrellensis(Raddi) Kuntze & Cachimbeiro & 3 & 66,67 & 3,57 & 4 & 1,22 & 0,02 & 0,93 & 5,73 \\
\hline Hancornia speciosaGomez & Mangaba & 2 & 66,67 & 3,57 & 3 & 0,82 & 0,02 & 0,64 & 5,03 \\
\hline Zanthoxylum riedelianumEngl. & $\begin{array}{l}\text { Mamica de } \\
\text { porca }\end{array}$ & 2 & 66,67 & 3,57 & 3 & 0,82 & 0,02 & 0,58 & 4,97 \\
\hline Malouetia cestroidesMüll. Arg. & Paineira & 3 & 33,33 & 1,79 & 4 & 1,22 & 0,03 & 1,06 & 4,07 \\
\hline Simarouba versicolorSt. Hil. X. & Mata cachorro & 2 & 33,33 & 1,79 & 3 & 0,82 & 0,01 & 0,54 & 3,15 \\
\hline $\begin{array}{l}\text { Simarouba opaca(Engl.) Radlk. ex } \\
\text { Engl. }\end{array}$ & Mata menino & 1 & 33,33 & 1,79 & 1 & 0,41 & 0,02 & 0,79 & 2,99 \\
\hline Stryphnodendron adstringens Mart. & Barbatimão & 1 & 33,33 & 1,79 & 1 & 0,41 & 0,01 & 0,53 & 2,73 \\
\hline Magonia pubescensA.St.-Hil. & Tingui & 1 & 33,33 & 1,79 & 1 & 0,41 & 0,01 & 0,53 & 2,73 \\
\hline Guarea guidonia $(\mathrm{L}$.) Sleumer & Marinheiro & 1 & 33,33 & 1,79 & 1 & 0,41 & 0,01 & 0,45 & 2,64 \\
\hline Copaifera langsdorffiiDesf. & Pau d'óleo & 1 & 33,33 & 1,79 & 1 & 0,41 & 0,01 & 0,34 & 2,54 \\
\hline $\begin{array}{l}\text { Aspidosperma cuspa(H.B.K.) } \\
\text { S.F.Blake. }\end{array}$ & Pereiro & 1 & 33,33 & 1,79 & 1 & 0,41 & 0,01 & 0,28 & 2,47 \\
\hline Guazuma ulmifoliaLam. & Mutamba & 1 & 33,33 & 1,79 & 1 & 0,41 & 0,01 & 0,26 & 2,45 \\
\hline Callisthene fasciculata(Spr.) Mart. & Carvoeiro & 1 & 33,33 & 1,79 & 1 & 0,41 & 0,01 & 0,24 & 2,43 \\
\hline $\begin{array}{lc}\text { TOTAL GERAL } \\
\end{array}$ & & $\overline{245}$ & $\overline{1866,67}$ & $\overline{100}$ & 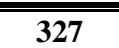 & $\overline{100}$ & $2, \mathbf{2 , 5 9}$ & $\overline{100}$ & 30 \\
\hline
\end{tabular}


As espécies encontradas correspondem a 2,4\% do total daquelas compiladas para a flora vascular do bioma Cerrado (MENDONÇA et al. 1998) e a 5\% das espécies relatadas por Castro et al. (1999) para a flora lenhosa dos cerrados do País.
O valor mais expressivo de DoR foi encontrado para a espécie Qualea parviflora, com 15,54\%, fato que pode ser explicado pelo alto número de indivíduos desta espécie na área amostrada. Os parâmetros fitossociológicos das dez espécies de maior V.I. podem ser observados na Figura 1.

Figura 1. Relação das dez espécies que apresentaram maiores índices de valor de importância (V.I.), ordenadas de forma decrescente, encontradas em área de cerrado sensu stricto , Paranã-TO.

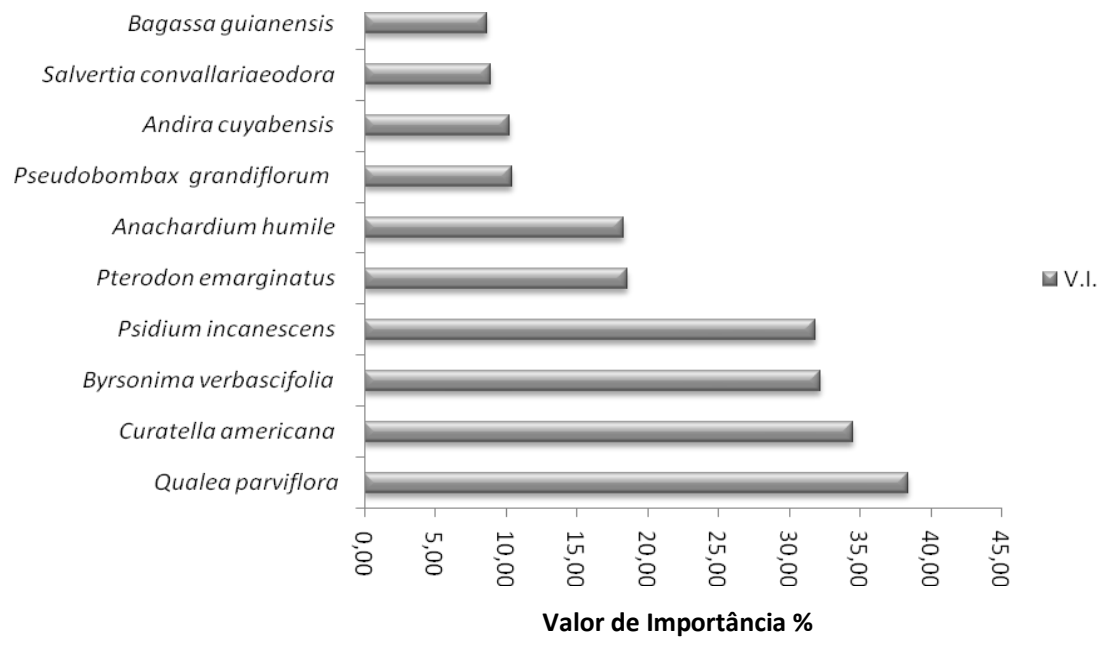

As espécies mais representativas quanto ao valor de importância também foram as que tiveram maior valor de cobertura, mantendo-se a mesma hierarquia. Esse resultado demonstra que a densidade e a dominância representaram eficientemente a estrutura da floresta analisada o que confirma o fato de que a frequência, de forma geral, está associada à densidade das espécies.

Em ecossistemas de floresta natural tropical, as espécies arbóreas podem apresentar diferentes tendências ou curvas de distribuição diamétrica. Diante do contexto foi possível observar que a menor classe diamétrica apresentou maior densidade de indivíduos, o que indica que a maioria das populações pode estar em fase inicial de estabelecimento (CARVALHO, 1982; 1992; SOUZA et al., 2010). A maior concentração de indivíduos nas primeiras classes de diâmetro pode caracterizar uma comunidade-estoque, o que é padrão em florestas tropicais estáveis com idade e composição de espécies variadas (SCOLFORO \& MELLO, 1997) (Figura 2).
As classes iniciais apresentaram maiores números de indivíduos, com a Classe $1(5,0-9,0 \mathrm{~cm})$ representando aproximadamente $58 \%$ do total amostrado e $45 \%$ superior a classe subsequente, sendo a redução progressiva do número de indivíduos por classes diamétricas.

À medida que aumenta o tamanho da classe diametrica a densidade de indivíduos diminui até atingir o seu menor índice na maior classe diamétrica, caracterizando uma curva do tipo exponencial ou denominada como "J" invertido (JESUS \& SOUZA, 1995); (SCOLFORO, 1998); (SOUZA et al., 2007).

A curva do tipo exponencial ou denominada como "J" invertido amostrada no presente trabalho segue os padrões verificados em estudos realizados por Felfili e Silva Júnior (2001) e Ferreira et al. (2009) em áreas de cerrado sensu stricto.

Figura 2. Distribuição diamétrica dos indivíduos encontrados em área de cerrado, município de Paranã-TO. Onde: Classe 1 5,0 à $9,0 \mathrm{~cm}$; Classe $2-9,1$ à 13,9 cm; Classe $3-14,0$ à 17,0 cm; Classe $4-17,1$ à 19,0 cm; Classe $5-19,1$ à 23,0 cm e Classe $6->23,1 \mathrm{~cm}$.

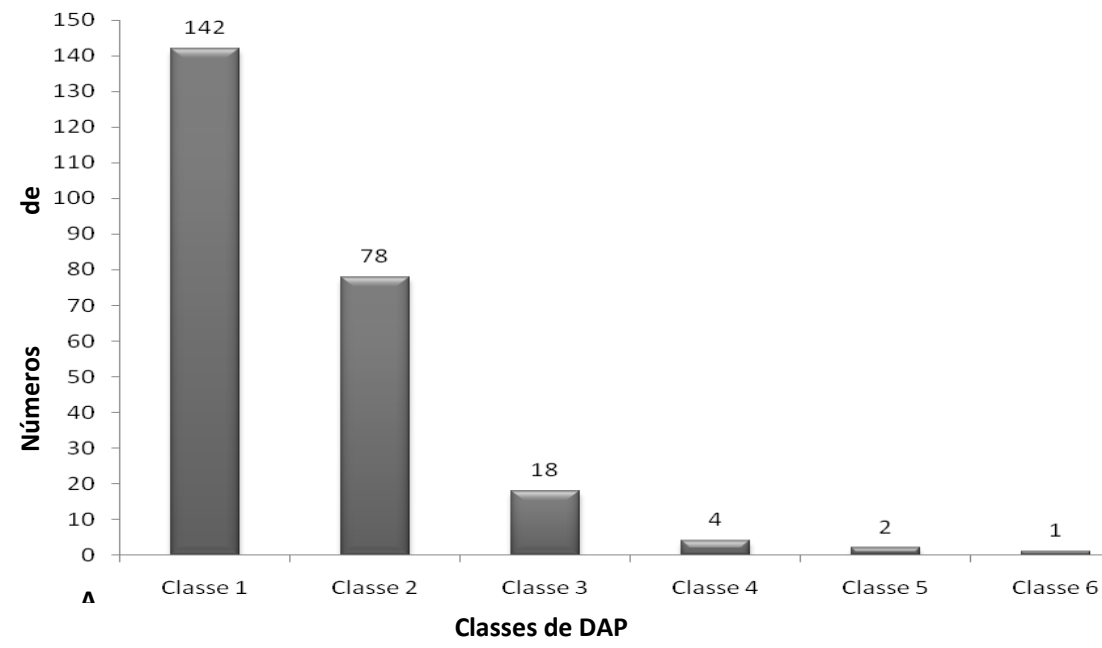


No presente trabalho cerca de $89 \%$ dos indivíduos amostrados apresentaram-se com diâmetros até $14,0 \mathrm{~cm}$ padrão confirmado no cerrado sensu stricto por (ASSUNÇÃO ; FELFILI, 2004).
Em relação à área basal das classes diamétricas pode-se perceber na figura 3, que as classes 1 e 2 se destacaram em relação as demais, coincidindo com as classes de maior número dos indivíduos amostrados estudados.

Figura 3. Distribuição da área basal encontrados em área de cerrado, município de Paranã-TO, nas classes diamétricas.

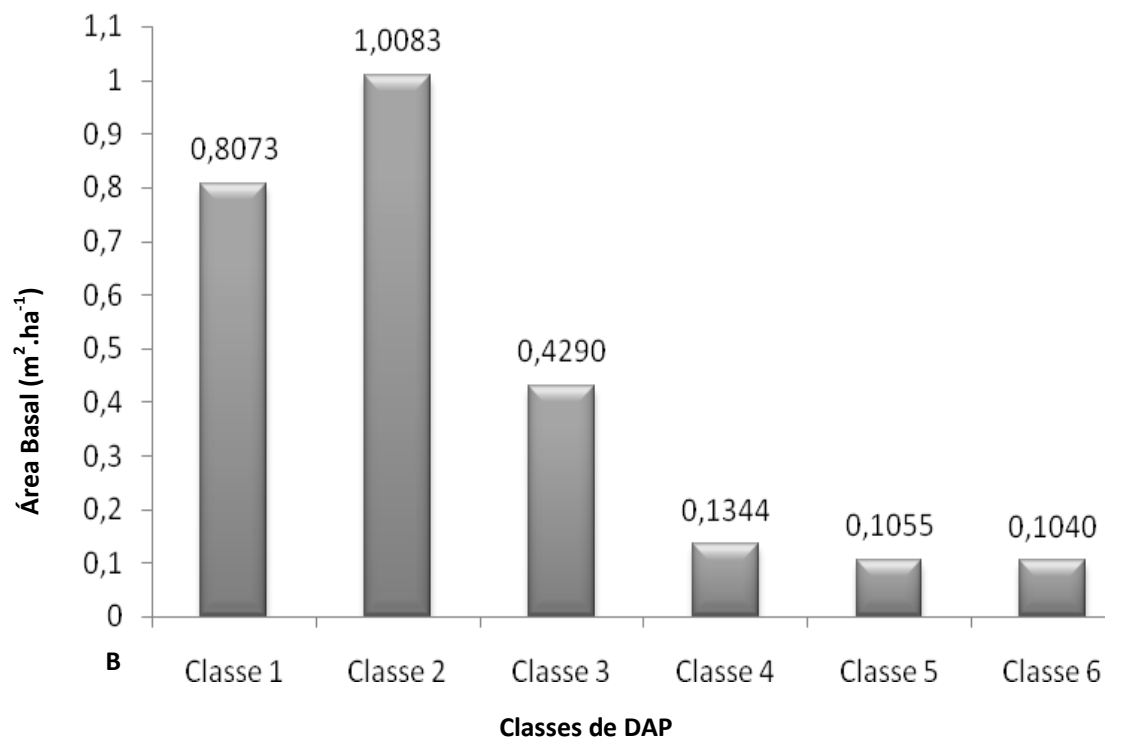

A relação hipsométrica do cerrado estudado mostrou que altura, sendo a redução gradativa a partir da primeira a maioria dos indivíduos se encontram nas primeiras classes a diamétrica (Figura 4).

Figura 4. Distribuição dos indivíduos encontrados em área de cerrado, município de Paranã-TO, em classes de altura. Onde: Classe 1 - 1,5 à 2,9 cm; Classe 2 - 3,0 a 4,4 cm; Classe $3-4,5$ a 5,9 cm; Classe 4 - 6,0 a 7,4 cm; Classe 5 - 7,5 a 8,9 cm; Classe $6-9,0$ a 10,4 e Classe $7->10,5$.

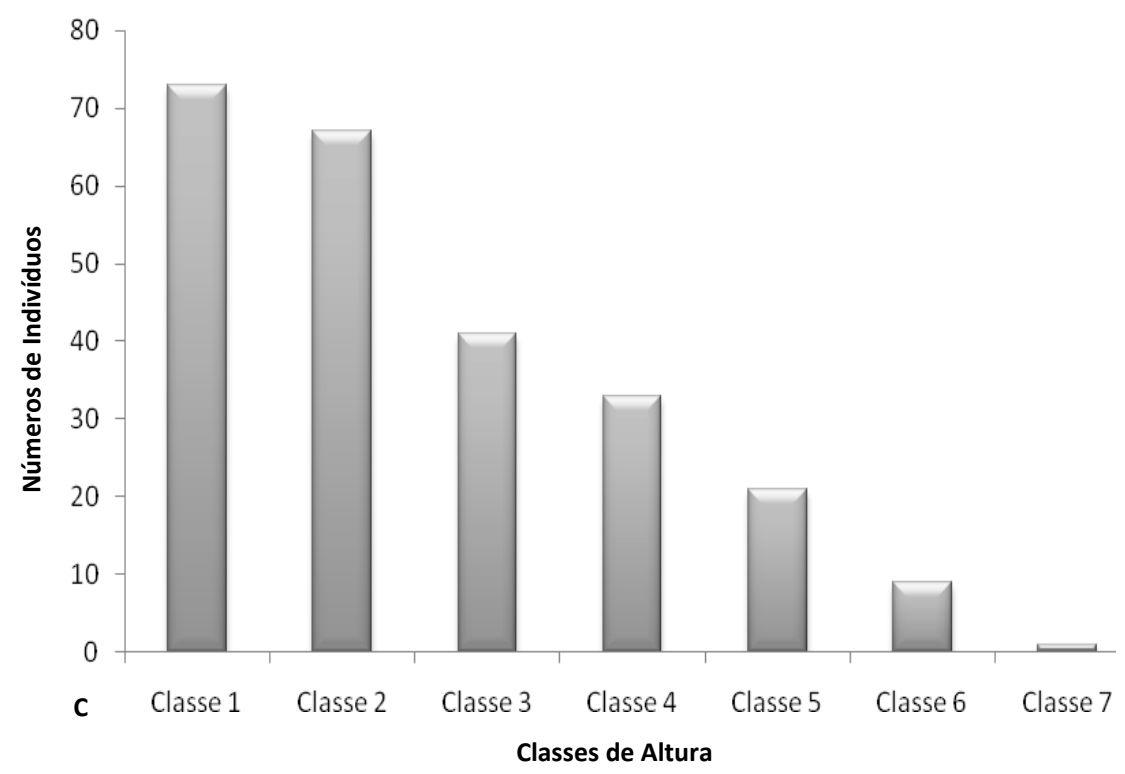

\section{CONCLUSÕES}

A composição florística da área estudada é coerente com as formações savânicas que predominam no bioma Cerrado do Brasil Central.

Qualea parviflora foi a espécie mais representativa dentro do componente arbóreo o que se conclui em altos valores de densidade e dominância nos estratos.
O cerrado sensu stricto estudado encontra-se em processo de sucessão inicial, caracterizado especialmente pela dinâmica da estrutura nos estratos inferiores.

\section{AGRADECIMENTOS}

A Universidade Federal do Tocantins - UFT, que através do Programa de Pós-Graduação em Ciências Florestais e Ambientais viabilizou a realização desse estudo. 


\section{REFERÊNCIAS BIBLIOGRÁFICAS}

ALHO, C.J.R. \& MARTINS, E.S. De Grão em Grão, o Cerrado Perde Espaço (Cerrado - Impactos do Processo de Ocupação). WWF-Fundo Mundial para a Natureza. Brasília. 1995.

APG III. 2009. An update of the Angiosperm Phylogeny Group classification for the orders and families of flowering plants: APG III. Botanical Journal of the Linnean Society 161: 105-121.

ASSUNÇÃO, S. L. \& FELFILI, J. M. Fitossociologia de umfragmento de cerrado sensu stricto na APA do Paranoá, DF,Brasil. Acta Botânica Brasil. 18(4): 903909. 2004.

BRASIL. MINISTÉRIO DO MEIO AMBIENTE. 2014. Biodiversidade brasileira: avaliação e identificação de áreasprioritárias para conservação, utilização sustentável erepartição de benefícios da biodiversidade brasileira. Brasília: MMA/SBF, 404p.

CARVALHO, J.O.P. Structure and dinamics of a logged over brazilian amazonian rain forest. Oxford: Oxford University, 1992. 215p. Tese (Doutorado em Filosofia) Oxford University, 1992.

CARVALHO, J. O. P. Análise estrutural da regeneração natural em floresta tropical densa na região do tapajós no estado do Pará. 1982. 128 f. Dissertação (mestrado em Engenharia Florestal) - Universidade Federal do Paraná, Curitiba, 1982.

CASTRO, A.A.J.F., MARTINS, F.R., TAMASHIRO, J.Y.\& SHEPHERD, G.J. 1999. How rich is the flora ofBrazilian cerrados? Annals of the Missouri Botanical Garden 86:192-226.

EITEN, G. The cerrado vegetation of Brazil. Botanical Review, v. 38, p.201-341, 1972.

EITEN, G. Vegetação. In: NOVAES PINTO, M. (Ed.). Cerrado: caracterização, ocupação e perspectiva. 2. ed. Brasília: UnB: SEMATEC,1994. p. 17-73.

FELFILI, J.M.; SILVA JÚNIOR, M.C. (orgs.). 2001.Biogeografia do Bioma Cerrado: estudo fitofisionômico da Chapada do Espigão Mestre do São Francisco.

FELFILI, J.M.; NOGUEIRA, P.E.; SILVA JÚNIOR, M.C.;MARIMON, B.S. \& DELITTI, W.B.C. 2002. Composiçãoflorística e fitossociologia do cerrado sensu stricto nomunicípio de Água Boa, MT. Acta Botanica Brasilica 16(1):103-112.

FERREIRA, M. J. et al. Avaliação da regeneração natural em nascentes perturbadas no município de Lavras, MG. Ciência Florestal, Santa Maria, v. 19, n. 2, p. 109-129, abr./jun. 2009.
FERREIRA, R. L. C.; SOUZA, A. L.; JESUS, R. M. de. 1998. Dinâmica da estrutura de uma floresta secundária de transição: II. Distribuição diamétrica. Revista Árvore, Viçosa, v. 22, n. 3,p. 331-344.

FERREIRA, R. L. C. Análise estrutural da vegetação da Estação Florestal de Experimental de Açu-RN, como subsídio básico para o manejo florestal. Viçosa: UFV, 1988. 90p. Dissertação (Mestrado em Ciência Florestal) - Universidade Federal de Viçosa, 1988.

FINOL, U.M. Nuevos parametros a considerar-se en el análisis estructural de las selvas virgenes tropicales. Rev. For. Venez. 14 (21): 29-42. 1971.

JESUS, R.M., SOUZA, A.L. Produção sustentável em mata secundária de transição: oito anos de monitoramento. Viçosa: SIF, 1995. 99p. (Documento SIF, 014).

KLINK, C.A. Relação entre o desenvolvimento agrícola e a biodiversidade. In:R.C. Pereira, L. C. B. Nasser (Eds.). Anais VIII Simpósio sobre o Cerrado, 1stInternational Symposium on Tropical Savanas - Biodiversidade e ProduçãoSustentável de Alimentos e fibras nos Cerrados. Embrapa CPAC. Brasília. Pg. 25-27. 1996.

LAMPRECHT, H. Ensayo sobre la estructura florística de la parte Sur-Oriental del Bosque Universitário "El Caimital"- Estado Barinas. Rev. For, Venez. 7 (1011):77-119. 1964.

LAMPRECHT, H. Ensayo sobre unos métodos para el análisis estructural de los bosques tropicales. Acta científica Venezolana, (2):57-65. 1962.

MACHADO, S. A.; BARTOSZECK, A. C. P. E S.; FILHO, A.F.; OLIVEIRA, E. B. 2006. Dinâmica da distribuição diamétricade bracatingais na Região metropolitana de Curitiba. Revista Árvore, Viçosa, v.30, n.5, p.759-768.

MARCUZZO, F. F. N.; GOULARTE, E. R. P.; MELO, D. C. R.; FILHO, R. F. P.; CARDOSO, M. R. D. 2011. Mapeamento espacial, temporal e sazonal das chuvas no bioma Cerrado do estado do Tocantins. Anais... XV Simpósio Brasileiro de Sensoriamento Remoto - SBSR, Curitiba, PR, Brasil, INPE p.5217.

MENDONÇA, R.; FELFILI, J. M.; WALTER, B. M. T.; SILVA JÚNIOR, M.C.; REZENDE, A. V.; FILGUEIRAS, T. S.; NOGUEIRA, P. E. N. Floravascular do Cerrado. In: SANO, S. M.; ALMEIDA, S. P. (Eds), Cerrado:ambiente e flora. Embrapa: Brasília - DF, 1998. p. 287-556.

MENDONÇA, R.C., FELFILI, J.M., WALTER, B.M.T.,SILVA JUNIOR, M.C., REZENDE, A.V., FILGUEIRAS,T.S. \& NOGUEIRA, P.E. 1998. Flora vascular docerrado. In Cerrado: ambiente e flora (S.M. Sano \&S.P. Almeida, eds.). Embrapa, Planaltina, p.289556. 
MITTERMEIER RA, ROBLES GIL P, MITTERMEIER CG. 1999. Hotspots: Earth's Biologically Richest and Most Endangered Terrestrial Ecoregions. CEMEX, Conservation International, and Agrupación Sierra Madre.

MUELLER-DOMBOIS D, ELLENBERG H. Aims and methods of vegetation ecology. New York: John Wiley \& Sons; 1974

MYERS N, MITTERMEIER RA, MITTERMEIER CG, DA FONSECA GAB, KENT J. 2000. Biodiversity hotpots for conservation priorities. Nature 403: 853-858.

OLIVEIRA, E. C. L. \& FELFILI, J. M. Estrutura e dinâmica da regeneração natural deuma mata de galeria no Distrito Federal, Brasil. Acta botanica brasílica. v. 19, n 4,p. 801-811, 2005.

OLIVEIRA-FILHO, A.T.; CARVALHO, D.A; VILELA E.A.; CURI, N.; FONTES,M.A.L.Diversity and structure of tree community of a fragment of tropical secondary Forestof Brazilian Atlantic Forest domain 15 and 40 years after logging. Revista brasileira de Botânica v. 27, n. 4, p. 685-701, 2004.

OLIVEIRA, M. C.; SCOLFORO, J. R. S.; MELLO, J. M.;OLIVEIRA, A. D.; ACERBI, F. W. 2006. Avaliação dediferentes níveis de intervenção na florística, diversidade esimilaridade de uma área de cerrado stricto sensu. Cerne,Lavras, v. 12, n. 4, p. 342-349.

PEREIRA-SILVA, E. F.L.; SANTOS, J. E.; KAGEYAMA, P. Y.; HARDT, E. 2004. Florística e fitossociologia dos estratosarbustivo e arbóreo de um remanescente de cerradão em umaUnidade de Conservação do Estado de São Paulo. Revista Brasil. Bot., V.27, n.3, p.533-544.

RIBEIRO, J. F.; WALTER, B. M. T. As principais Fitofisionomias do BiomaCerrado. In: SANO. S. M.; ALMEIDA, S. P.; RIBEIRO, J.F. Cerrado: ecologia e flora. Embrapa: Brasília - DF, 2008. 1.279p.

RIBEIRO, J. F.; WALTER, B. M. T. Fitofisionomias do Bioma Cerrado. In:SANO, S. M.; ALMEIDA, S. P. Cerrado: ambiente e flora. Embrapa:Brasília - DF, 1998. $556 \mathrm{p}$.
RIZZO, J.A. Flora do estado de Goiás: Coleção Rizzo. Ed Universidade Federalde Goiás. Goiânia-GO. 1981, 35p.

SCOLFORO, J. R. S. 1998. Manejo florestal. Lavras: UFLA/FAEPE, 438 p.

SCOLFORO, J. R. S.; MELLO, J. M. Inventário florestal. Textos Acadêmicos. Lavras: UFLA/FAEPE, 1997. $341 \mathrm{p}$.

SEPLAN-TO. Secretaria do Planejamento e Orçamento do Tocantins. Acessado em 23/07/2015. Disponível em: http://web.seplan.to.gov.br/Arquivos/Publicacoes/Atlas2 012/z_Completo_Atlas_do_Tocantins_2012_portugues. pdf.

SILVA， L. O.; COSTA， D. O.; SANTO-FILHO, K. E.;FERREIRA， H. D.; BRANDÃO， D. 2002. Levantamentoflorístico e fitossociológico em duas áreas de Cerrado sensu stricto no Parque Estadual da Serra de Caldas Novas, Goiás. Acta Botânica Brasilica, São Paulo, v. 16, n. 2, p. 43-53.

SOUZA, A. L. et. al. Caracterização fitossociológica em áreas de ocorrência natural de candeia (Eremanthus erythropappus (d.c.) (Macleish). Árvore, Viçosa, MG, v. 31, n. 4, p. 667-667, 2007.

SOUZA, G. S. et. al. Krigagem ordinária e inverso do quadrado da distância aplicados na espacialização de atributos químicos de um argissolo. Scientia Agraria, Curitiba, v. 11, n. 1, p. 73-81, jan./fev. 2010.

SIMINSKI, A.; MANTOVANI, M.; REIS, M. S.; FANTINI, A.C. 2004. Sucessão florestal secundária no município de SãoPedro de Alcântara, litoral de Santa Catarina: estrutura ediversidade. Ciência Florestal, Santa Maria, v. 14 , n. 1 , p. 21-33.

WALTER, R. M. T.; GUARINO, E. S. G. Comparação do método deparcelas com o "levantamento rápido" para amostragem da vegetaçãoarbórea do Cerrado sensu stricto. Acta Botanica Brasílica, São Paulo,v. 20, n. 2, p. 285-298, 2006. 